\title{
Escala Clance do Fenômeno do Impostor: Adaptação Brasileira
}

\author{
Thereza Christina Garcia Bezerra ${ }^{1}$ \\ Larisse Helena Gomes de Macêdo Barbosa \\ Katia Correa Vione ${ }^{3}$ \\ Rebecca Alves Aguiar Athaydet \\ Valdiney Veloso Gouveia ${ }^{5}$ \\ ${ }^{1}$ Centro Universitário de Patos, Patos, Paraíba, Brasil \\ ${ }^{2}$ Universidade Cruzeiro do Sul, São Paulo, São Paulo, Brasil \\ ${ }^{3}$ University of Derby, Derby, Derbyshire, United Kingdom \\ ${ }^{4}$ Faculdade de Ciências Médicas, João Pessoa, Paraíba, Brasil \\ ${ }^{5}$ Universidade Federal da Paraíba, João Pessoa, Paraíba, Brasil
}

\begin{abstract}
Resumo
A Síndrome do Importor (SI) reflete dúvidas sobre conquistas e capacidades pessoais na ausência de evidências externas que reforcem tal percepção. Este estudo objetivou adaptar ao contexto brasileiro a Escala Clance do Fenômeno do Impostor (ECFI), reunindo evidências de sua validade fatorial e consistência interna. Dois estudos foram realizados. No Estudo 1, participaram 201 estudantes universitários $\left(M_{\text {idade }}=22,5, D P=5,04 ; 71,6 \%\right.$ mulheres) e, no Estudo 2, participaram 252 estudantes de pós-graduações $\left(M_{\text {iddade }}=30,4 \mathrm{DP}=6,18 ; 75 \%\right.$ mulheres). Todos os participantes responderam a ECFI e perguntas demográficas. Análises fatoriais exploratórias (AFEs) foram realizadas em ambos os estudos, indicando uma estrutura unifatorial, que explicou entre 46,3\% (Estudo 1) e 57\% (Estudo 2) da variância total, apresentando consistência interna (alfa de Cronbach) acima de 0,90. Concluiu-se que a ECFI é uma medida psicometricamente adequada, que poderá ser usada em estudos futuros para compreender a dimensão geral da síndrome do impostor e seus correlatos.
\end{abstract}

Palavras-chave: síndrome do impostor, escala, validade, precisão

\section{Clance Impostor Phenomenon Scale:}

Brazilian adaptation

\begin{abstract}
The impostor syndrome reflects doubts about personal achievements and abilities in the absence of external evidence that supports such perception. This study aimed to adapt the Clance Impostor Phenomenon Scale (CIPS) to the Brazilian context, gathering evidence of its factorial validity and reliability. Two studies were conducted. Study 1 included 201 undergraduate students $\left(\mathrm{M}_{\text {age }}=22.5, \mathrm{SD}=5.04 ; 71.6 \%\right.$ female $)$ and Study 2 analyzed 252 graduate students $\left(\mathrm{M}_{\text {age }}=30.4, \mathrm{SD}=6.18 ; 75 \%\right.$ female $)$. All participants answered the CIPS and demographic questions. Exploratory factor analyses (EFA) were conducted in both studies, indicating a one-factor structure, explaining between $46.3 \%$ (Study 1) and 57\% (Study 2) of the total variance, showing internal consistency (Cronbach's alpha) above 0.90 . The CIPS proved to be a psychometrically adequate scale that could be used in future studies to understand the overall dimension of the impostor syndrome and its correlates.

Keywords: Impostor Syndrome; Scale; Validity; Reliability.
\end{abstract}

\section{Escala Clance del Fenómeno del Impostor:}

Adaptación brasileña

\section{Resumen}

El síndrome del impostor (SI) refleja dudas sobre los logros y capacidades personales en la ausencia de evidencias externas que refuerzan esta percepción. Este estudio tuvo como objetivo adaptar la Escala Clance del Fenómeno del Impostor (ECFI) al contexto brasileño, reuniendo evidencias de su validez factorial y consistencia interna. Dos estudios fueron realizados. En el Estudio 1 participaron 201 estudiantes universitarios $\left(M_{\text {edad }}=22.5, D S=5.04 ; 71.6 \%\right.$ mujeres $)$ y 252 estudiantes de postgrado $\left(M_{\text {edad }}\right.$ $=30.4, D S=6.18 ; 75 \%$ mujeres) en el Estudio 2. Todos los participantes respondieron la ECFI y preguntas demográficas. Se realizaron análisis factoriales exploratorios (AFE) en los dos estudios, lo que indica una estructura unifactorial que explica entre el 46.3\% (Estudio 1) y el 57\% (Estudio 2) de la varianza total, mostrando una fiabilidad (alfa de Cronbach) superior a .90. En conclusión, la ECFI es una medida psicométricamente adecuada, que puede ser utilizada en futuros estudios para comprender la dimensión general del síndrome del impostor y sus correlatos. Palabras clave: Síndrome del Impostor; Escala; Validez; Fiabilidad. 


\section{Introdução}

O conceito de Síndrome do Importor (SI), também conhecido como fenômeno do impostor, foi introduzido por Clance e Imes (1978) para descrever percepções e sentimentos de ser um presumível impostor, alguém que duvida de suas próprias conquistas mesmo com evidências contrárias a essa rotulação. Quem apresenta esse tipo de percepção, pensamento ou sentimento de ser um impostor atribui êxitos a fatores exteriores e não às próprias habilidades e aos seus esforços (Simon \& Choi, 2018). Dessa forma, os "impostores" são incapazes de receber elogios ou reconhecimentos acerca de suas conquistas; quando estes têm lugar, causam-lhes grau elevado de ansiedade, estresse e falta de autoconfiança (Clance \& Imes, 1978).

Inicialmente, a SI foi identificada em um grupo de mulheres de sucesso que demonstravam possuir crenças distorcidas de que não mereciam o que haviam alcançado, acreditando que seu sucesso resultava de interpretações distorcidas de suas habilidades ou eram meramente decorrentes de sorte. De fato, elas se consideravam uma fraude, julgando que não mereciam as conquistas alcançadas (Parkman, 2016). Além disso, indivíduos que experimentam a sensação de serem impostores têm medo de que pessoas relevantes em suas vidas percebam que eles são uma fraude, aspecto que afeta negativamente sua saúde mental. Nessa direção, os impostores geralmente sabotam seu sucesso, de modo que novas possibilidades de crescimento passam a ser recusadas, pois evitam por receio de serem descobertos como tais, isto é, percebem-se como verdadeiramente impostores. Dessa forma, esquivam-se ou se mantêm distantes de todas as atividades sociais, passando a viverem confinados em grupos menores ou mesmo isolados (Cowman \& Ferrari, 2002).

Segundo Clance e Imes (1978), existem quatro comportamentos que influenciam diretamente a SI, fazendo com que o próprio indivíduo se sabote. O primeiro diz respeito à realização de trabalhos árduos, de modo que os outros não percebam sua incapacidade e incompetência; o segundo está relacionado à falsidade, fazendo com que use ideias opostas às suas, pois acredita que se usasse verdadeiramente as suas não se daria bem; o terceiro se refere ao uso de fascínio e simpatia para atrair os seus superiores, passando a acreditar que o seu sucesso se deve a essa relação; e, por último, trata-se de conduta de evitação, fugindo do sucesso para escapar de ações rudes e desprezo dos demais, priorizando ser bem aceito socialmente.
A Síndrome do Impostor costuma ter lugar no decorrer da vida acadêmica, podendo permear e influenciar a carreira profissional. Dessa forma, pode afetar negativamente o desempenho acadêmico ou profissional, além de ter implicações na saúde mental das pessoas acometidas. Contudo, também pode variar segundo características demográficas. Por exemplo, Villwock, Sobin, Koester e Harris (2016) observaram níveis mais elevados de Síndrome do Impostor em estudantes não brancos, do sexo feminino e no último ano do curso. De forma semelhante, Cokley et al. (2017) observaram que o impostorismo atua como moderador da relação entre preconceito percebido e indicadores de saúde mental (e.g., depressão, ansiedade) em estudantes negros, asiátios e latino-americanos nos Estados Unidos.

Em razão do anteriormente comentado, torna-se evidente que a Síndrome do Impostor leva a cognições e comportamentos disfuncionais que, por sua vez, estão relacionados com bem-estar psicológico e desempenho reduzidos (Badawy, Gazdag, Bentley, \& Brouer, 2018; Neureiter \& Traut-Mattausch, 2016; Schubert \& Bowker, 2017; Villwock, Sobin, Koester, \& Harris, 2016). Para que os efeitos dessa síndrome possam ser reconhecidos e que fatores de proteção ou tratamento possam ser explorados, é essencial contar com um instrumento com propriedades psicométricas aceitáveis. Sendo assim, foram realizadas buscas na literatura em relação a instrumentos para avaliar a Síndrome do Impostor no contexto brasileiro. Concretamente, em 10 de fevereiro de 2018, foram realizadas buscas em três bases de dados (Google Acadêmico, SciELO e Lilacs), utilizando os seguintes termos em Português "Escala Impostor", "Escala Impostorismo", "Escala Síndrome do Impostor" e "Escala do Fenômeno Impostor". Nenhum artigo foi encontrado, o que motivou nova busca com esses mesmos termos em inglês; então, identificaram-se 13 artigos que tratavam do tema, porém não apresentavam qualquer medida que tivesse sido adaptada ao contexto brasileiro. Dessa forma, passou-se a considerar a literatura internacional, identificando as medidas disponíveis e escolhendo a que parecesse mais psicometricamente apropriada à realidade brasileira, empreendendo esforços para adaptá-la.

\section{Medindo a Sindrome do Impostor}

Os instrumentos para medir a síndrome do impostor são facilmente encontrados na literatura internacional [e.g., Impostor Phenomenon (IP; Clance \& Imes, 1978), Harvey Impostor Phenomenon Scale (HIPS; Harvey 
\& Katz, 1985), Impostor Test (IT; Clance, 1985), Perceived Frandulence Scale (PFS; Kolligian \& Sternberg, 1991)]. Embora essas medidas estejam comumente correlacionadas entre elas (Leary, Patton, Orlando, \& Funk, 2000), algumas reúnem muitos itens (e.g., a PFS se compõe de 51 itens), enquanto outras apresentam problemas psicométricos (e.g., HIPS), como inadequação de itens (Hellman \& Caselman, 2004) ou coeficientes baixos de consistência interna (Chrisman, Pieper, Clance, Holland, \& Glickauf-Hughes, 1995).

Considerando o anteriormente comentado, decidiu-se ter em conta como objeto deste estudo uma medida relativamente breve, formada por 20 itens, mas que vem apresentando propriedades psicométricas satisfatórias: a Clance Impostor Phenomenon Scale (CIFS), em português Escala Clance do Fenômeno do Impostor (ECFI; Chrisman et al., 1995). Esta tem sido amplamente usada em diversos contextos, como acadêmico (Bernard, Dollinger, \& Ramanjah, 2002) e profissional (Haney, Birkholz, \& Rutledge, 2018; Leach, Nygaard, Chipman, Brunsvold, \& Marek, 2019). Ademais, a ECFI parece ser mais sensível para diferenciar "impostores" de "não impostores" (Holmes, Kertay, Adamson, Holland \& Clance, 1993). Portanto, procura-se descrevê-la a seguir.

A ECFI foi desenvolvida por Pauline R. Clance no contexto canadense. Seus 20 itens procuram medir características definidoras da Síndrome do Impostor, como medo de ser incapaz de repetir o sucesso, medo de ser avaliado e medo de ser menos capaz do que os outros (Chrisman et al., 1995). Esses itens são pontuados em escala de 5 pontos, devendo a pessoa indicar o quanto cada um a descreve. Essa autora criou o instrumento com o objetivo de avaliar se as pessoas possuíam os medos de fracassarem, serem avaliadas, não repetirem o sucesso e não serem reconhecidas (Langford \& Clance, 1993). Inicialmente, resultados da análise fatorial exploratória indicaram uma solução composta por três fatores (Chrisman et al., 1995). Porém, French (2008) realizou uma análise confirmatória com uma amostra de doutorandos, observando que um modelo composto por dois fatores explicaria melhor a estrutura da medida, propondo sua versão reduzida, formada por 16 itens.

Apesar dos achados prévios, estudos mais recentes com a ECFI vêm demonstrando, por meio de análises fatoriais confirmatórias que testam diferentes modelos, que a estrutura fatorial mais adequada compreende aquela de um único fator (Jöstl, Bergsmann, Lüftenegger, Schober, \& Spiel, 2012; Simos \& Choi, 2018). O modelo unifatorial oferece a vantagem da parcimônia, ademais de reunir indicadores de ajuste aceitáveis, conforme tais estudos; por exemplo, os itens dessa escala apresentaram saturações elevadas, variando entre 0,48 e 0,82 , tendo o fator geral coeficientes alfas de Cronbrach superiores a 0,70. Portanto, trata-se de uma escala psicometricamente adequada para avaliar a Síndrome do Impostor, o que motivou adaptá-la ao contexto brasileiro, reunindo evidências de sua validade fatorial e consistência interna. Descrevem-se a seguir os estudos realizados.

\section{Estudo 1. Escala Clance do Fenômeo Impostor: Análises Exploratórias}

Conforme indicado anteriormente, a Escala do Fenômeno Impostor de Clance (EFIC) se configura como um instrumento psicometricamente adequado para avaliar a Síndrome do Impostor. Apesar da relevância desse construto, que pode contribuir para entender a percepção que as pessoas têm de competência limitada e o fracasso que disso pode decorrer, incluindo suas repercussões negativas na saúde mental (e.g., ansiedade, baixa autoestima, depressão), ainda não se encontrou qualquer medida a respeito no contexto brasileiro. Portanto, realizou-se essa primeira tentativa de adaptar a EFIC, procurando conhecer sua estrutura fatorial e consistência interna.

\section{Método}

\section{Participantes}

O presente estudo contou com a participação de 201 estudantes universitários de instituições privadas $(87,6 \%)$ e públicas $(12,4 \%)$, com idade média de $22,5(D P=5,04$; variando de 17 a 47 anos). A maioria era mulher $(71,6 \%)$, solteira $(71,4 \%)$ e de classe social média $(52,2 \%)$, tendo nível mediano de religiosidade (40,3\%). Tratou-se de uma amostra de conveniência, participando aqueles que, estando devidamente matriculados, concordaram em voluntariamente fazer parte do estudo.

\section{Instrumentos}

Os participantes responderam questões sociodemográficas (sexo, idade, estado civil, classe socioeconômica e religiosidade), dispostas no final de questionário, além da Escala Clance do Fenômeno do Impostor (Clance, 1985). Esse instrumento, originalmente desenvolvido em língua inglesa, teve sua adaptação autorizada para este estudo, sendo traduzido 
para o português por meio do procedimento de back translation, contando com a participação de dois psicólogos bilíngues; as versões finais foram avaliadas por um terceiro psicólogo igualmente bilíngue, que atestou a adequação de sua tradução. Mantiveram-se seus 20 itens originais (e.g., Mesmo quando obtenho alguma conquista e sou reconhecido (a) por isso, tenho dúvidas se sou capaz de repetir o sucesso; Às vezes sinto que meu sucesso foi devido a algum tipo de sorte), respondidos em escala do tipo Likert, de 5 pontos, variando de 1 (Não me descreve) a 5 (Descreve-me totalmente). Essa versão foi submetida à validação semântica, considerando dez estudantes universitários de cidade do interior da Paraíba, verificando-se a compreensão dos itens, da escala de resposta e das instruções sobre como respondê-los. Nenhuma alteração foi exigida, resultando na versão que poderá ser disponibilizada ao leitor interessado.

\section{Procedimento}

A coleta de dados foi realizada na plataforma Google Docs e por meio de questionários impressos. Os respondentes foram recrutados por meio de redes sociais (e.g., Whats App, Facebook, Instagram), disponibilizando-se o link para acessar ao questionário ou foram recrutados em ambiente universitário, quando se tratou de versão lápis e papel. Em ambas as versões foram descritos os objetivos da pesquisa, o procedimento envolvido e destacada a importância da participação, que deveria ser voluntária, assegurando-se o anonimato. Os respondentes tiveram que concordar (pesquisa on-line) ou assinar (questionário impresso) Termo de Consentimento Livre e Esclarecido, estando cientes de que poderiam se retirar da pesquisa a qualquer momento sem ônus, consoante com o que determina a Resolução no 510/2016, do Conselho Nacional de Saúde. O projeto foi aprovado pelo Comitê de Ética em Pesquisa com Seres Humanos, seguindo-se as recomendações vigentes (parecer de número 88272218.6.0000.518). Em média, 20 minutos foram suficientes para concluir a participação no estudo.

\section{Análises de Dados}

Para análise dos dados, utilizou-se o programa Factor (versão 10.9.02; Lorenzo-Seva \& Ferrando, 2006), realizando-se uma análise fatorial exploratória (EFA). Esta foi feita com a matriz de correlações policóricas, levando em consideração o nível de medida ordinal característico do instrumento, bem como da violação da hipótese de normalidade multivariada dos dados
(Holgado-Tello, Chacón-Moscoso, BarberoGarcia, \& Vila-Abad, 2010). Adotou-se o método de extração Robust Unweighted Least Squares (RULS). O procedimento de análises paralelas (Optimal implementation of Parallel Analysis - PA) foi utilizado para a retenção de fatores, considerando-se sua robustez (Lorenzo-Seva, Timmerman, \& Kiers, 2011).

\section{Resultados}

O índice Kaiser-Meyer-Olkin (KMO) foi igual a 0,92 e o Teste de Esfericidade de Bartlett, igual a $\chi^{2}(190)$ $=1.848,20, \operatorname{com} p<0,001$, indicando que a matriz de dados era passível de fatoração. O procedimento de PA sugeriu um fator como a solução mais representativa dos dados [RMSEA $=0,056$, NNFI $=0,99$, CFI $=0,99$ e WRMR $=0,07)$. A partir da AFE, todos os itens carregaram significativamente no fator geral, explicando 46,3\% da variância total; suas saturações são apresentadas na Tabela 1. Estabeleceu-se saturação mínima de 0,30 para definir a pertença do item ao fator, constatando-se a relevância de todos eles, cujas saturações variaram de 0,33 (item 1) a 0,87 (item 15). O alfa foi de 0,91 .

Estudo 2. Escala Clance do Fenômeo do Impostor: Replicação da Estrutura

Assim como o estudo anterior, este se configura como de natureza psicométrica. Especificamente, objetivou checar a estrutura fatorial da Escala Clance do Fenômeno do Impostor, avaliando se a solução unifatorial poderia emergir ou ser replicada em uma amostra de pós-graduandos. Nesse caso, optou-se por uma análise fatorial exploratória (AFE) tendo em conta que se pretendeu replicar os achados prévios, algo que tem sido recomendado por Osborne e Fitzpatrick (2012). A propósito, Thompson (2004) também recomenda replicar achados de análise fatorial exploratória de instrumentos em fase de elaboração ou adaptação, checando se a estrutura emerge em amostras distintas.

\section{Método}

\section{Participantes}

Participaram deste estudo 252 estudantes de mestrado (36\%) ou doutorado $(64 \%)$ de várias universidades, predominantemente as públicas $(80 \%)$. Estes possuíam média de idade de 30,4 anos ( $D P=6,18)$, a maioria do sexo feminino $(75 \%)$ e solteira $(53 \%)$, declarando nível médio de religiosidade $(M=2,6, D P=1,19$; 
Tabela 1.

Estrutura Fatorial da Escala Clance do Fenômeno Impostor

\begin{tabular}{|c|c|c|c|}
\hline \multirow{2}{*}{ Item } & \multirow{2}{*}{ Conteúdo } & \multicolumn{2}{|c|}{ Estudos } \\
\hline & & 1 & 2 \\
\hline 15 & $\begin{array}{l}\text { Mesmo quando obtenho alguma conquista e sou reconhecido (a) por isso, tenho } \\
\text { dúvidas se sou capaz de repetir o sucesso. }\end{array}$ & 0,87 & 0,90 \\
\hline 13 & $\begin{array}{l}\text { Às vezes tenho medo de que os outros descubram o quanto de conhecimento ou } \\
\text { habilidade realmente me falta. }\end{array}$ & 0,79 & 0,87 \\
\hline 14 & $\begin{array}{l}\text { Geralmente temo que eu possa falhar em uma nova tarefa ou empreendimento } \\
\text { embora eu faça bem aquilo que tento. }\end{array}$ & 0,78 & 0,85 \\
\hline 04 & $\begin{array}{l}\text { Quando as pessoas me elogiam por algo que eu realizei, temo que não serei capaz } \\
\text { de corresponder às suas expectativas em relação a mim no futuro. }\end{array}$ & 0,75 & 0,84 \\
\hline 06 & $\begin{array}{l}\text { Eu tenho medo de que as pessoas importantes pra mim possam descobrir que eu } \\
\text { não sou tão capaz quanto elas pensam que sou. }\end{array}$ & 0,75 & 0,88 \\
\hline 16 & $\begin{array}{l}\text { Se recebo muitos elogios e reconhecimento por algo que realizei, tendo a } \\
\text { desconsiderar a importância do que fiz. }\end{array}$ & 0,75 & 0,79 \\
\hline 17 & $\begin{array}{l}\text { Frequentemente comparo minha habilidade com aqueles que me cercam e acho } \\
\text { que eles possam ser mais inteligentes do que eu. }\end{array}$ & 0,74 & 0,79 \\
\hline 18 & $\begin{array}{l}\text { Às vezes me preocupo em não conseguir me sair bem em um projeto ou em um } \\
\text { exame/prova, embora outras pessoas próximas tenham confiança que eu vou me } \\
\text { sair bem. }\end{array}$ & 0,76 & 0,81 \\
\hline 12 & $\begin{array}{l}\text { Às vezes sinto-me decepcionado(a) com minhas realizações atuais e acho que } \\
\text { deveria ter realizado muito mais. }\end{array}$ & 0,73 & 0,71 \\
\hline 07 & $\begin{array}{l}\text { Eu tendo a me lembrar de situações nas quais eu não fiz meu melhor mais do que } \\
\text { situações em que fiz meu melhor. }\end{array}$ & 0,68 & 0,80 \\
\hline 10 & É difícil para mim aceitar elogios sobre minha inteligência ou minhas realizações. & 0,68 & 0,77 \\
\hline 08 & Eu raramente faço um projeto ou tarefa tão bem quanto eu gostaria de fazer. & 0,64 & 0,70 \\
\hline 20 & $\begin{array}{l}\text { Eu me sinto mal e desencorajado se eu não for "o melhor" ou pelo menos "muito } \\
\text { especial" em situações que envolvam realizações. }\end{array}$ & 0,64 & 0,59 \\
\hline 03 & Eu evito avaliações se possível e tenho pavor de ser avaliado(a). & 0,59 & 0,68 \\
\hline 09 & $\begin{array}{l}\text { Às vezes eu sinto ou acredito que meu sucesso (na vida pessoal, trabalho ou } \\
\text { estudos) é resultado de algum tipo de erro. }\end{array}$ & 0,67 & 0,84 \\
\hline 11 & Às vezes sinto que meu sucesso foi devido a algum tipo de sorte. & 0,62 & 0,87 \\
\hline 05 & $\begin{array}{l}\text { Às vezes, acho que obtive minha posição atual ou obtive meu sucesso atual porque } \\
\text { estava no lugar certo na hora certa ou conhecia as pessoas certas. }\end{array}$ & 0,46 & 0,76 \\
\hline 02 & $\begin{array}{l}\text { Eu posso dar a impressão de que eu sou mais competente do que eu realmente } \\
\text { sou. }\end{array}$ & 0,42 & 0,54 \\
\hline 01 & $\begin{array}{l}\text { Muitas vezes tive sucesso em um teste ou tarefa mesmo com medo de não me sair } \\
\text { bem antes de entendê-la. }\end{array}$ & 0,33 & 0,46 \\
\hline 19 & $\begin{array}{l}\text { Se eu estou para receber uma promoção no trabalho ou ser reconhecido por algo, } \\
\text { eu hesito em falar para as outras pessoas até que isso de fato aconteça. }\end{array}$ & 0,45 & 0,37 \\
\hline \multicolumn{2}{|c|}{ Número de itens } & 20 & 20 \\
\hline \multicolumn{2}{|c|}{ Valor próprio } & 9,3 & 11,5 \\
\hline \multicolumn{2}{|c|}{ \% Variância explicada } & 46,3 & 57,0 \\
\hline \multicolumn{2}{|c|}{ Alfa de Cronbach } & 0,91 & 0,95 \\
\hline
\end{tabular}


escala variando de $1=$ Nada religioso a $5=$ Totalmente religioso). Tratou-se de amostra de conveniência, tendo participado pessoas que se disponibilizaram a fazê-lo de forma voluntária.

\section{Instrumentos e Procedimento}

Os participantes responderam aos mesmos instrumentos do Estudo 1, isto é, perguntas sociodemográficas (e.g., sexo, idade e classe social) e a Escala Clance do Fenômeno do Impostor. Tratou-se de ampliação de projeto anteriormente aprovado pelo Comitê de Ética, sendo a coleta de dados realizada por meio de um questionário on-line desenvolvido no Google Docs. O recrutamento dos participantes ocorreu por meio das redes sociais (Whats App, Facebook, Instagram) e contatos pessoais dos pesquisadores, encaminhando o link para que acessassem o questionário. Descreveram-se os objetivos da pesquisa, o procedimento envolvido, a possibilidade de se retirar do estudo sem ônus e a natureza voluntária da participação, assegurando-se o anonimato. Prévia a participação, os estudantes precisavam ler e concordar com o Termo de Consentimento Livre e Esclarecido.

\section{Análise de Dados}

Utilizou-se o programa Factor (versão 10.9.02; Lorenzo-Seva \& Ferrando, 2006) para realizar a análise fatorial exploratória (EFA), considerando-se a matriz de correlações policóricas, como ocorreu no estudo anterior (Holgado-Tello et al., 2010). Igualmente, adotou-se o método de extração Robust Unweighted Least Squares (RULS), assumindo o procedimento de análises paralelas (Optimal implementation of Parallel Analysis - PA) para retenção de fator (Lorenzo-Seva, Timmerman, \& Kiers, 2011).

\section{Resultados}

Inicialmente, realizou-se a análise paralela, que demonstrou ser mais adequada à solução de um fator. Posteriormente, checou-se a adequação da amostra para efetuar uma análise fatorial exploratória (KMO $=0,94$; Teste de Esfericidade de Bartlett, $\chi^{2}(190)=$ $3.473,90, p<0,001$; RMSEA $=0,065, \mathrm{NNFI}=0,99$, $\mathrm{CFI}=0,99$ e WRMR $=0,07)$. Utilizando como ponto de corte cargas fatoriais maiores que 0,30 , observou-se que todos os itens saturaram satisfatoriamente no fator, cujas cargas variaram entre 0,37 (item 19) e 0,90 (item 15). Essa solução unifatorial explicou $57 \%$ da variância total, apresentando alfa de Cronbach de 0,95. Na última coluna da Tabela 1 são descritos mais detalhadamente esses resultados.

\section{Discussão}

O presente artigo teve como objetivo adaptar a Escala Clance do Fenômeno do Impostor, reunindo evidências de sua validade fatorial e consistência interna. Nesse sentido, contou-se com a participação de estudantes de graduação e pós-graduação (mestrado ou doutorado), realizando-se dois estudos de natureza psicométrica que, no geral, atestaram a adequação dessa medida como unifatorial. Portanto, confia-se que esse objetivo tenha sido alcançado.

A Escala Clance do Fenômeno do Impostor é composta por 20 itens que avaliam a Síndrome do Impostor. A partir dos dois estudos, foi possível encontrar uma solução unifatorial dessa medida, que não apoiou sua versão original em língua inglesa, pressupunha três fatores: medo de avaliação, medo de não repetir o sucesso e medo de ser menos capaz que os outros (Clance, 1985). Não obstante, em outros estudos, estruturas diferentes têm também sido observadas, como as unifatorial (Jöstl et al., 2012; Simon \& Choi, 2018) e bifatorial (French, Ullrich-French \& Follman, 2008). Quanto ao modelo unifatorial especificamente, Jöstl, Bergsmann, Lüftenegger, Schober e Spiel (2012) o identificaram em uma amostra de doutorandos; Simon e Choi (2018), por outro lado, testaram três modelos em uma amostra de doutorandos: unifatorial, bifatorial e trifatorial, sendo que o bifatorial e o trifatorial apresentaram correlações fortes entre seus fatores, endossando a escolha do modelo unifatorial, que se revelou mais adequado, reunindo itens com cargas fatoriais entre 0,48 e 0,82 , contando com alfa de Cronbach acima de 0,80.

Esses resultados, que evidenciam a adequação de uma estrutura unifatorial, foram similares aos observados no presente estudo, que, inclusive, foram mais promissores, não se limitando a estudantes de doutorado. Concretamente, consideraram-se amostras de graduandos, mestrandos e doutorandos, constatando a possibilidade de extrair um único fator geral de Síndrome do Impostor, cujos itens apresentaram saturações superiores ao que se recomenda na literatura (0,30; Pasquali, 2010), tendo valores médios superiores a 0,50 . Comprovadamente, essa estrutura unifatorial se replicou, apresentando coeficiente de congruência fatorial acima de 0,90 , que tem sido recomendado na literatura (Gorsuch, 1983). 
O parâmetro de consistência interna (alfa de Cronbach) da Escala Clance do Fenômeno do Impostor foi bastante promissor considerando o ponto de corte da literatura (0,70; Clark \& Watson, 1995; Tabachnick \& Fidell, 2013). De fato, observaram-se coeficientes inclusive superiores aos observados por Simon e Choi (2018). Entretanto, o teste de Hakstian-Whalen $\left(\mathrm{M}_{\mathrm{H}-\mathrm{W}}\right.$; Kim \& Feldt, 2008) indicou que a consistência interna foi estatisticamente superior no Estudo 2 quando comparado ao Estudo 1. Ressalta-se, não obstante, que esse resultado pode se dever às características dos participantes do Estudo 2: eram profissionais graduados, matriculados em cursos de mestrado ou doutorado, diversos já sendo professores universitários. Desse modo, tratava-se de pessoas mais maduras (maior média de idade), além de presumivelmente lidarem mais diretamente com questões envolvendo o binômio fracasso-sucesso, sendo comumente cobradas por suas realizações, o que pode tornar mais saliente a Síndrome do Impostor. Essa situação tem potencial de aumentar a consistência das respostas das pessoas, tornando o alfa de Cronbach maior do que se esperaria para jovens estudantes de graduação (Gouveia, Santos, \& Milfont, 2009).

Mesmo alcançando os objetivos propostos no presente estudo, não se podem descartar possíveis limitações. No caso, há que se assinalar que as amostras consideradas foram de conveniência (não aleatórias). Isso impede generalizar os achados quanto à presença da Síndrome do Impostor no contexto brasileiro, não sendo possível inclusive para o cenário específico em que foram obtidos os dados. Porém, deve-se ponderar que o propósito não foi conhecer o quantitativo de pessoas que apresentavam essa síndrome, mas conhecer a estrutura e a consistência interna da medida, i.e., da ECFI. Portanto, as amostras consideradas podem ser consideradas suficientes (Tabachnick \& Fidell, 2013).

Não obstante, ao anteriormente comentado, muito ainda precisará ser conhecido sobre a Escala Clance do Fenômeno do Impostor. Por exemplo, a qualidade dos itens poderá ser aferida em termos de discriminação e endosso, considerando abordagem da Teoria de Resposta ao Item (TRI; Pasquali, 2020). Será preciso também verificar a estrutura unifatorial no contexto brasileiro, contrastando-a com modelos bi e trifatoriais, realizando análises fatoriais confirmatórias (Simon \& Choi, 2018). Seria importante, ainda, conhecer evidências validade discriminante dessa medida, por exemplo, com desejabilidade social, um construto que pode enviesar as respostas das pessoas (Almiro, 2017), que tenderiam a negar que vivenciam essa síndrome.
Por fim, recomenda-se avaliar a estabilidade temporal dessa medida, referendando seu parâmetro psicométrico de precisão.

Em resumo, apesar de existem maneiras variadas de estudar a Síndrome do Impostor, as publicações nacionais acerca desse construto ainda são escassas. $\mathrm{O}$ presente artigo representa uma contribuição por oferecer as primeiras evidências de adequação psicométrica da Escala Clance do Fenômeno do Impostor. Isso, seguramente, poderá estimular novas pesquisas que procurem conhecer seus antecedentes e consequentes, embasando ações futuras que pretendam promover mudanças na percepção que as pessoas têm delas mesmas, evitando sua própria sabotagem e prejuízos à sua saúde mental.

\section{Referências}

Almiro, P. A. (2017). Uma nota sobre a desejabilidade social e o enviesamento de respostas. Avaliação Psicológica, 16(3). doi: 10.15689/ap.2017.1603.ed

Badawy, R. L., Gazdag, B. A., Bentley, J. R., \& Brouer, R. L. (2018). Are all impostors created equal? Exploring gender differences in the impostor phenomenon-performance link. Personality and Individual Differences, 131, 156-163. doi: 10.1016/j. paid.2018.04.044

Bernard, N. S., Dollinger, S. J., \& Ramanjah, N. V. (2002). Applying the big five personality factors to the impostor phenomenon. Journal of Personality Assessment, 78(2), 321-333. doi: 10.1207/ S15327752JPA7802_07

Brasil (2016). Resolução no 510/16 do Conselho Nacional de Saúde, de 07 de abril de 2016. Dispõe sobre diretrizes e normas regulamentadoras de pesquisas envolvendo seres humanos. Ministério da Saúde, Brasil. Recuperado de https://bvsms.saude.gov.br/bvs/ saudelegis/cns/2013/res0466_12_12_2012.html

Chrisman, S. M., Pieper, W. A., Clance, P. R., Holland, C. L., \& Glickauf-Hughes, C. (1995). Validation of the Clance Impostor Phenomenon Scale. Journal of Personality Assessment, 65(3), 456-467. doi: 10.1207/ s15327752jpa6503_6

Clance, P. R. (1985). The impostor phenomenon: Overcoming the fear that haunts your success. Atlanta, GA: Peachtree.

Clance, P., R., \& Imes, S. (1978). The imposter phenomenon in high achieving women: Dynamics 
and therapeutic intervention. Psychotherapy Theory, Research and Practice, 15(3), 1-8. doi: 10.1037/ h0086006

Clark, L. A., \& Watson, D. (1995). Constructing validity: Basic issues in objective scale development. Psychological Assessment, 7(3), 309-319. doi: 10.1037/1040-3590.7.3.309

Cokley, K., Smith, L., Bernard, D., Hurst, A., Jackson, S., Stone, S., ... \& Roberts, D. (2017). Impostor feelings as a moderator and mediator of the relationship between perceived discrimination and mental health among racial/ethnic minority college students. Journal of Counseling Psychology, 64(2), 141-154. doi: 10.1037/ cou0000198

Cowman, S., \& Ferrari, J. (2002). "Am I for real?" Predicting impostor tendencies from self-handicapping and affective components. Social Behavior and Personality: An International Journal, 30, 119-126. doi: 10.2224/sbp.2002.30.2.119

French, B. F., Ullrich-French, S. C., \& Follman, D. (2008). The psychometric properties of the Clance Impostor Scale. Personality and Individual Differences, 44(5), 1270-1278. doi: 10.1016/j.paid.2007.11.023

Gorsuch, R. (1983). Factors analysis. (2a ed.). Lawrence Erlbaum Assosicates.

Gouveia, V. V., Santos, W. S., \& Milfont, T. L. (2009). O uso da estatística na avaliação psicológica: Comentários e considerações práticas. Em C. S. Hutz (Eds.), Avanços e polêmicas em avaliação psicológica: Em homenagem a Jurema Alcides Cunba (pp. 127-155). São Paulo: Casa do Psicólogo.

Haney, T. S., Birkholz, L., \& Rutledge, C. (2018). A workshop for addressing the impact of the imposter syndrome on clinical nurse specialists. Clinical Nurse Specialist, 32(4),189-194. doi: 10.1097/ NUR.0000000000000386

Harvey, J. C., \& Katz, C. (1985). If I'm so successful, why do I feel like a fake?: The impostor phenomenon. New York: St. Martin's Press.

Hellman, C. M., \& Caselman, T. D. (2004). A psychometric evaluation of the Harvey Impostor Phenomenon Scale. Journal of Personality Assessment, 83(2),161-166.doi:10.1207/s15327752jpa8302_10

Holgado-Tello, F., Chacón-Moscoso, S., Barbero-García, I., \& Vila-Abad, E. (2010). Polychoric versus Pearson correlations in exploratory and confirmatory factor analysis of ordinal variables. Quality and Quantity, 44(1), 153-166. doi: 10.1007/ s11135-008-9190-y

Holmes, S. W., Kertay, L., Adamson, L. B., Holland, C. L., \& Clance, P. R. (1993). Measuring the impostor phenomenon: A comparison of Clance's IP Scale and Harvey's IP Scale. Journal of personality assessment, 60(1), 48-59.

Jöstl, G., Bergsmann, E., Lüftenegger, M., Schober, B., \& Spiel, C. (2012). When will they blow my cover? The impostor phenomenon among Austrian doctoral students. Zeitschrift für Psychologie, 220(2), 109-120. doi: 10.1027/2151-2604/ a000102

Kim, S., \& Feldt, L. S. (2008). A comparison of tests for equality of two or more independent alpha coefficients. Journal of Educational Measurement, 45(2), 179-193.

Kolligian, J., Jr., \& Sternberg, R. J. (1991). Perceived fraudulence in young adults: Is there an "impostor syndrome?” Journal of Personality Assessment, 56, 308-326. doi: 10.1207/s15327752jpa5602_10

Langford, J., \& Clance, P. R. (1993). The imposter phenomenon: Recent research findings regarding dynamics, personality and family patterns and their implications for treatment. Psychotherapy: Theory, Research, Practice, Training, 30(3), 495-501. doi: 10.1037/0033-3204.30.3.495

Leach, P. K., Nygaard, R. M., Chipman, J. G., Brunsvold, M. E., \& Marek, A. P. (2019). Impostor phenomenon and burnout in general surgeons and general surgery residents. Journal of SurgicalEducation, 76(1), 99-106. doi: 10.1016/j.jsurg.2018.06.025

Leary, M. R., Patton, K., Orlando, A., \& Funk, W. W. (2000). The impostor phenomenon: Self-perceptions, reflected appraisals, and interpersonal strategies. Journal of Personality, 68, 725-756. doi: 10.1111/1467-6494.00114

Lorenzo-Seva, U., \& Ferrando, P. J. (2006). FACTOR: A computer program to fit the exploratory factor analysis model. Behavioral Research Methods, Instruments and Computers, 38(1), 88-91. doi: 10.3758/ BF03192753

Lorenzo-Seva, U., Timmerman, M. E., \& Kiers, H. A. L. (2011). The Hull Method for selecting the number of common factors. 
Multivariate Behavioral Research, 46(2), 340-364. doi: 10.1080/00273171.2011.564527

Neureiter, M., \& Traut-Mattausch, E. (2016). An inner barrier to career development: Preconditions of the impostor phenomenon and consequences for career development. Frontiers in psychology, 7, 48. doi: 10.3389/fpsyg.2016.00048

Osborne, J. W., \& Fitzpatrick, D. C. (2012). Replication analysis in exploratory factor analysis: What it is and why it makes your analysis better. Practical $A s-$ sessment, Research \& Evaluation, 17, 1-8. Recuperado de https://scholarworks.umass.edu/cgi/viewcontent.cgi? article $=1283 \&$ context $=$ pare

Parkman, A. (2016). The imposter phenomenon in higher education: Incidence and impact. Journal of Higher Education Theory and Practice, 16(1), 5161. Recuperado de http://www.na-businesspress. com/JHETP/ParkmanA_Web16_1_.pdf

Pasquali, L. (2020). TRI-Teoria de resposta ao item: Teoria, procedimentos e aplicacões. Curitiba, PR: Editora Appris.

Schubert, N., \& Bowker, A. (2017). Examining the impostor phenomenon in relation to self-esteem level and self-esteem instability. Current Psychology, 1-7. doi: 10.1007/s12144-017-9650-4

Simon, M., \& Choi, Y. J. (2018). Using factor analysis to validate the Clance Impostor Phenomenon Scale in sample of science, technology, engineering and mathematics doctoral students. Personality and Individual Differences, 121, 173-175. doi: 10.1016/j. paid.2017.09.039

Tabachnick, B. G., \& Fidell, L. S. (2013). Using multivariate statistics ( $\left.6^{\mathrm{a}} \mathrm{ed}.\right)$. London: Pearson.

Thompson, B. (2004). Exploratory and confirmatory factor analysis: Understanding concepts and applications. American Psychological Association.

Villwock, J. A., Sobin, L. B., Koester, L. A., \& Harris, T. M. (2016). Impostor syndrome and burnout among american medical students: A pilot study. International journal of medical education, 7, 364-369. doi: 10.5116/ijme.5801.eac4

Recebido em: 07/10/2019

Reformulado em: 01/05/2020

Aprovado em: 09/06/2020 


\section{Anexo \\ Escala Clance do Fenômeno Impostor (Adaptação Brasileira)}

INSTRUÇÕES. A seguir, encontra-se uma lista com 20 afirmações. Não existem respostas certas ou erradas, apenas gostaríamos de saber a sua opinião, o quanto você concorda ou discorda com cada afirmação.

\begin{tabular}{ccccc}
\hline $\mathbf{1}$ & $\mathbf{2}$ & $\mathbf{3}$ & $\mathbf{4}$ & $\mathbf{5}$ \\
\hline Não me descreve & Raramente & Às vezes & Frequentemente & Me descreve totalmente \\
\hline
\end{tabular}

1. ___ Muitas vezes tive sucesso em um teste ou tarefa mesmo com medo de não me sair bem antes de entendê-la.

2. ___ Eu posso dar a impressão de que eu sou mais competente do que eu realmente sou.

3. ___ Eu evito avaliações se possível e tenho pavor de ser avaliado (a).

4. ___ Quando as pessoas me elogiam por algo que eu realizei, temo que não serei capaz de corresponder às suas expectativas em relação a mim no futuro.

5. ___ As vezes, acho que obtive minha posição atual ou obtive meu sucesso atual porque estava no lugar certo na hora certa ou conhecia as pessoas certas.

6. ___ Eu tenho medo que as pessoas importantes pra mim possam descobrir que eu não são tão capaz quanto elas pensam que sou.

7. ___ Eu tendo a me lembrar de situações nas quais eu não fiz meu melhor mais do que situações em que fiz meu melhor.

8. ___ Eu raramente faço um projeto ou tarefa tão bem quanto eu gostaria de fazer.

9. ___ Às vezes eu sinto ou acredito que meu sucesso (na vida pessoal, trabalho ou estudos) é resultado de algum tipo de erro.

10. ___ É difícil para mim aceitar elogios sobre minha inteligência ou minhas realizações.

11. ___ Às vezes sinto que meu sucesso foi devido a algum tipo de sorte.

12. ___ Às vezes sinto-me decepcionado (a) com minhas realizações atuais e acho que deveria ter realizado muito mais.

13. ___ Às vezes tenho medo de que os outros descubram o quanto de conhecimento ou habilidade realmente me falta.

14. ___ Geralmente temo que eu possa falhar em uma nova tarefa ou empreendimento embora eu geralmente faça bem aquilo que tento.

15. ___ Mesmo quando obtenho alguma conquista e sou reconhecido (a) por isso, tenho dúvidas se sou capaz de repetir o sucesso.

16. ___ Se recebo muitos elogios e reconhecimento por algo que realizei, tendo a desconsiderar a importância do que fiz.

17. ___ Frequentemente comparo minha habilidade com aqueles que me cercam e acho que eles possam ser mais inteligentes do que eu.

18. ___ As vezes me preocupo em não conseguir me sair bem em um projeto ou em um exame/prova, embora outras pessoas próximas tenham confiança que eu vou me sair bem.

19. ___ Se eu estou para receber uma promoção no trabalho ou ser reconhecido por algo, eu hesito em falar para as outras pessoas até que isso de fato aconteça.

20. ___ Eu me sinto mal e desencorajado se eu não for "o melhor" ou pelo menos "muito especial” em situações que envolvam realizações. 
Sobre os autores:

Thereza Christina Garcia Bezerra é docente e Psicóloga Organizacional e do Trabalho no Centro Universitário de Patos (UNIFIP) e Mestranda em Psicologia Social pela Universidade Federal da Paraíba (UFPB), onde é colaboradora do Laboratório de Psicologia da Mídia (LPM). Graduada em Psicologia e Especialista em Avaliação Psicológica (UNIFIP, 2019; 2020) e Especialista em Psicologia Organizacional e do Trabalho (FAVENI, 2020). Possui interesse principalmente nas Áreas de Pesquisa Quantitativa, Análise de Dados, Psicologia Organizacional e do Trabalho, Psicologia Social, Avaliação Psicológica, Síndrome do Impostor e Validação/Adaptação de instrumentos. Foi monitora das disciplinas de Filosofia, Psicometria, Técnica de Análise de Dados e Análise Experimental.

ORCID: https://orcid.org/0000-0002-9014-5697

E-mail: tchristinapsico@gmail.com

Larisse Helena Gomes de Macêdo Barbosa é Doutora e Mestre em Psicologia Social pela Universidade Federal da Paraíba, Graduada em Psicologia na mesma universidade. Membro Colaborador do núcleo de pesquisa Bases Normativas do Comportamento Social (BNCS). Atualmente é professora da Universidade Cruzeiro do Sul, São Paulo. Tem trabalhado, principalmente, nas áreas de Pesquisa, Análise de Dados, Psicologia Social, Psicologia Positiva e Avaliação Psicológica.

ORCID: https://orcid.org/0000-0002-2965-5540

E-mail: larissehelena@hotmail.com

Katia Correa Vione é Doutora em Psicologia pela Cardiff University, Mestra em Psicologia Social e Graduada em Psicologia pela Universidade Federal da Paraíba. É colaboradora do grupo de Estudos e Práticas em Psicologia Positiva (GEPPsi+) da Universidade Federal do Espírito Santo e do Núcleo de Avaliação Psicológica em Saúde (NapsiS) da Universidade Federal do Ceará. Possui interesse nas áreas de Psicometria, Psicologia Social, Avaliação Psicológica, Metodologia e Estatística e Psicologia Positiva. Atualmente é professora da University of Derby.

ORCID: https://orcid.org/0000-0001-6633-8520

E-mail: katiavione@gmail.com

Rebecca Alves Aguiar Athayde é Doutora e Mestre em Psicologia Social pela Universidade Federal da Paraíba. Graduada em Psicologia pela mesma instituição, recebeu os prêmios de Láurea acadêmica e Jovem Pesquisador. Membro colaborador do BNCS. Atualmente, é professora da Faculdade de Ciências Médicas, estando trabalhado, principalmente, nas áreas de Pesquisa e Análise de Dados. É parecerista do SATEPSI e da Revista Temas em Psicologia.

ORCID: https://orcid.org/0000-0002-0776-9985

E-mail: rebeccaathayde@gmail.com

Valdiney Veloso Gouveia é Graduado em Psicologia (Universidade Federal da Paraíba, UFPB) e Direito (Centro Universitário de João Pessoa), Especialista em Psicometria e Mestre em Psicologia Social e do Trabalho (Universidade de Brasília) e Doutor em Psicologia Social (Universidade Complutense de Madri). É Professor Titular de Psicologia Social na UFPB e Pesquisador 1A do CNPq. Seus interesses de pesquisa se centram nas áreas de Psicologia Social e Avaliação Psicológica, pesquisando sobre valores humanos, bem-estar subjetivo e comportamentos pró e antissociais. ORCID: https://orcid.org/0000-0003-2107-5848

E-mail:vvgouveia@gmail.com

Contato com os autores:

tchristinapsico@gmail.com

larissehelena@hotmail.com

katiavione@gmail.com

rebeccaathayde@gmail.com

vvgouveia@gmail.com

Psico-USF, Bragança Paulista, v. 26, n. 2, p. 333-343, abr./jun. 2021 